\title{
Inez Maus (2020). Kompetenzmanual Autismus (KOMMA). Praxisleitfaden für den Bildungs-, Wohn- und Arbeitsbereich
}

\section{Stuttgart: Verlag W. Kohlhammer, ISBN 978-3-17-035669-6, 124 Seiten, $36 €$}

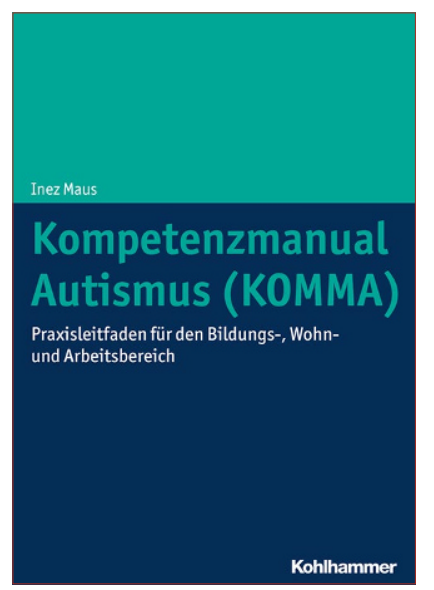

Nachdem Inez Maus im Jahr 2017 „Geschwister von Kindern mit Autismus" veröffentlichte, folgt nun mit „Kompetenzmanual Autismus (KOMMA)“ ihr viertes Buch. Der sehr praxisorientierte Titel legt diesmal den Schwerpunkt auf Unterstützungsund Fördermöglichkeiten für Menschen mit Autismus im Bereich Bildung, Wohnen und Arbeit. Um überhaupt Förderung und Unterstützung zu ermöglichen, braucht es zunächst ein grundlegendes Wissen zum Thema Autismus und ein Verständnis für die autismustypischen Besonderheiten. Dem widmet sich die Autorin nach einer einführenden Beschreibung ihres Manuals sehr umfassend und anschaulich in den ersten zwei Kapiteln. Sie geht dabei auf aktuelle Forschungen und Erkenntnisse ebenso ein wie auch auf geschichtliche Zusammenhänge. Darüber hinaus fließen eigene Beobachtungen und Erfahrungen ein, und sie lässt ihren autistischen Sohn zu Wort kommen. Das macht das Buch insgesamt sehr authentisch.

Autismus ist nicht heilbar. Insofern liegt es nahe, dass Hilfen und Unterstützung über alle Lebenskontexte von Menschen mit Autismus hinweg und über die gesamte Lebensspanne zu leisten sind. Und sie müssen sich nach den individuellen Bedürfnissen ausrichten, das heißt im „Einklang mit der autistischen Natur des Empfängers stehen “ (S. 29), wie es die Autorin formuliert. Insofern werden im dritten Kapitel die autismustypischen Besonderheiten sehr anschaulich beschrieben und mit praktischen Empfehlungen unterlegt. Mit Blick auf die Wahrnehmungsbesonderheiten des autistischen Störungsbildes braucht es immer wieder Übersetzungen, damit sich Autisten in der nicht-autistischen Alltagsund Lernwelt sicher orientieren können. Das Kompetenzmanual ist ein Beispiel dafür, wie Unterstützung aussehen und organisiert werden kann. Nicht zuletzt geht es insgesamt um die Schaffung eines gemeinsamen Raumes zur Verständigung und es geht um den Abbau von Barrieren. Gerade die im Anhang befindlichen Bearbeitungsbögen und Pfadanalysen machen sensibel dafür, wie notwendig es für Menschen mit Autismus ist, ein dem Störungsbild entsprechendes Umfeld zu schaffen. Gerade Lebensbereiche mit vielen Optionen, wie Freizeitgestaltung, Auswahl von Speisen usw. sind für Menschen mit Autismus oft schwer zu meistern. Hier brauchen sie Zeit zur Entscheidungsfindung, Visualisierungshilfen und oftmals zusätzliche Informationen, um, wie Inez Maus hervorhebt, Überlastung und Stress zu vermeiden. Dennoch kommt es gerade in neuen und reizüberflutenden Situationen zu Krisen und Belastungsreaktionen, weil Menschen mit Autismus nur unzureichend spontan einen eigenen Handlungsplan abrufen können. Eine sorgfältige Analyse stressauslösender Reize und mögliche Erklärungen sowie Handlungsvorschläge sind ebenso im Buch zu finden, wie Ideen für eine möglichst unabhängige Alltags- und Lebensführung.

So ist das Kompetenzmanual nicht nur im Sinne einer Förderung und Vermittlung hin zur autistischen Zielgruppe zu verstehen, sondern mit dem im Buch vorgestellten Material wird ein gegenseitiges Lernen möglich. 